\title{
Was lässt das Geräusch im Ohr verstummen?
}

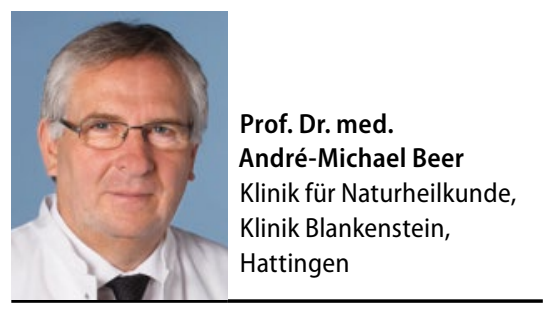

\author{
Ohrgeräusche sind für die Betroffenen quälend und therapeutisch schwer zu beeinflussen. \\ Prof. A.-M. Beer macht jedoch Hoffnung: Er berichtet über gute naturheilkundliche \\ Behandlungsmöglichkeiten, vor allem bei chronisch therapieresistentem Tinnitus.
}

\begin{abstract}
Phytopharmaka
Phytotherapeutisch können Ginkgo-biloba-Präparate eingesetzt werden. Ginkgoblätter verbessern nachweislich die Mikrozirkulation, sie hemmen die Thrombozytenaggregation und die Erythrozytenadhäsion. Die Erythrozytenflexibilität wird gesteigert, und die Blutviskosität sinkt.

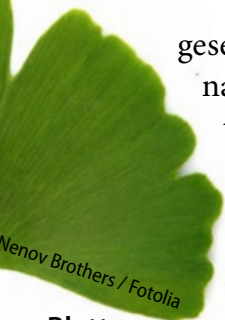

Ginkgo-Blatt

gesetzt werden [2]. Die Einnahme kann postoperativ wieder aufgenommen werden.

Ginkgo-biloba-

Präparate werden zur symptomatischen

Therapie des Tinnitus in einer Dosierung von $2 \times 120 \mathrm{mg} / \mathrm{Tag}$, morgens und abends 1 Filmtablette, für mindestens 8 Wochen empfohlen.
\end{abstract} Nachgewiesen wurden weiterhin die Inaktivierung von Sauerstoffradikalen, der Membranschutz durch Hemmung der Radikal-induzierten Lipidperoxidation, die Stabilisierung der Mitochondrienmembran, eine Verbesserung der Glukose- und Sauerstoffutilisation, eine Erhöhung energiereicher Phosphate und eine beschleunigte Rückbildung eines ischämischen, metabolischen und posttraumatischen Hirnödems. Die Wirksamkeits-mitbestimmenden Inhaltsstoffe sind Flavonglykoside, Terpenlactone (Ginkgolide A, B, C, Bilobalid) und $<5$ ppm Ginkgolsäure.

Als Nebenwirkungen können leichte Magen-Darm-Beschwerden, Kopfschmerzen, Schwindel und allergische Hautreaktionen auftreten. In vereinzelten Fallberichten kam es unter Dauermedikation mit Ginkgo-biloba-Extrakten $\mathrm{zu}$ einer erhöhten intraoperativen Blutungsneigung und zu zerebralen Blutungen. Auch eine Wirkverstärkung von gerinnungshemmenden Substanzen wird postuliert. Eine präoperative laborchemische Kontrolle der Blutgerinnung ist deshalb obligat. Ginkgo-biloba-Extrakte sollten drei Tage präoperativ ab-

\section{Aderlass und Blutegel}

Als hypovolämische Hämodilutionstherapie hat der Aderlass Einlass in die konventionelle Medizin gefunden, etwa bei Polyzythämie und Hämochromatose. Naturheilkundlich wird meist eine isobzw. hypervolämische Hämodilutionstherapie durchgeführt, bei der die zuerst abgelassene Blutmenge dann durch eine Infusionslösung (z. B. $\mathrm{NaCl}$ 0,9\%) wieder ausgeglichen wird.

Erfahrungsgemäß bringt auch eine Blutegelbehandlung - je 2 Blutegel beidseits über den Mastoidknochen - eine deutliche Besserung der Tinnitusbeschwerden.

Neuraltherapeutisch sind Injektionen mit Procain an das Mastoid und in das Ganglion stellatum möglich.

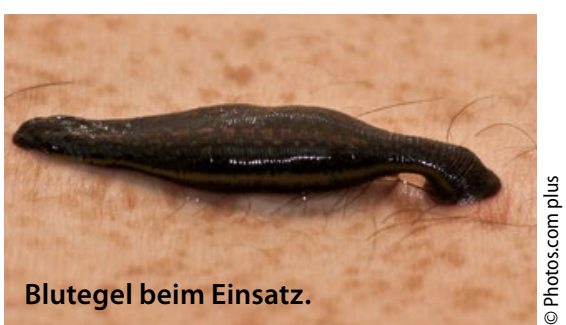

\section{Ordnungstherapie}

Oft sind dauerhafte psychisch-physische Belastungen eine wesentliche Mitursache des chronisch rezidivierenden Tinnitus und können eine psychotherapeutische Behandlung nötig machen. In vielen Fällen kann aber auch durch einfache ordnungstherapeutische Analysen und Beratungen zur Lebensweise sowie Entspannungsverfahren eine Besserung erreicht werden.

\section{Kieferorthopädische Maßnahmen}

Kiefergelenk, Zähne, Kaumuskulatur und Halswirbelsäule sind eng miteinander verknüpft. Durch anhaltende Verspannungen der Kaumuskulatur kann ein Tinnitus entstehen, da das Kiefergelenk gegen das Innenohr einen erhöhten Druck ausübt. Auch aufgrund von Fehlstellungen im Bereich des Kiefers kann sich ein chronischer Tinnitus entwickeln. Manche Patienten neigen unter Stress zu nächtlichem Zähneknirschen, das den Kiefer belastet. Eine Okklusionsanalyse zeigt, ob etwa ein Fehlbiss besteht. Fehlstellungen können mithilfe von Aufbissschienen oder anderen Spezialschienen zur Bissführung oder einer Korrektur der Bissebenen ausgeglichen werden.

Zusätzlich können spezielle krankengymnastische oder manualtherapeutische Maßnahmen verordnet werden.

$\rightarrow$ Literatur

1. Beer, A.-M., M. Adler (Hrsg). Leitfaden Naturheilverfahren für die ärztliche Praxis. Elsevier, Urban \& Fischer, München, 2011

2. Beer A.-M. et al. MMW Fortsch. Med. 2016 158(15):72-75 